\title{
In-situ Atomic-scale Imaging of Unidirectional Oxide Growth During the Oxidation of Metals
}

Guangwen Zhou

SUNY Binghamton University, Binghamton, New York, United States

The spontaneous formation of oxide nanowires (or whiskers) from oxidation of metals is a wellestablished phenomenon dating back to 1950s [1]. However, the microscopic mechanism underlying the one-directional oxide growth has been a topic of controversy over several decades. Several mechanisms (e.g., evaporation and condensation [2], short-circuit diffusion [3]) based on different observations have been proposed, however, no proposed models are capable of explaining all key features of oxide whiskering including the driving force, microstructure evolution, and one-dimensional growth. From a fundamental point of view, the oxide whisker formation in the oxidation of metals is a very interesting and important problem, because it requires an understanding of the coupling between materials kinetic processes (such as mass transport, crystal growth, phase transformation), chemistry (solid-state reaction), and mechanics of stress generation and relaxation within the growing oxide.

More recently, the extreme environments of high-temperature oxidation have received increasing attention for reactive assembly of novel oxide nanostructures. Particularly, a notable effort has been directed to oxide nanowire growth by thermal oxidation of metals $[4,5]$, largely due to its technical simplicity and large-scale growth capability. This requires deeper fundamental knowledge of the oxide growth mechanism for manipulation of the growth process to control size, shape, defects, therefore functionalities of the oxides. Growth kinetics plays a key role in controlling morphology, shape, and facets of nanocrystals. In bulk or ensemble systems, crystal growth proceeds through the nucleation and growth processes and the kinetic understanding is developed around the statistical averaging of these two convoluted processes. Thus, many key aspects of the crystal growth may be buried in these entangled events and cannot be resolved directly. Among the leading challenges is the ability to disentangle these events via visualizing complex growth processes under in situ/operando conditions. Transmission electron microscopy (TEM) has evolved dramatically in recent years and the development of environmental TEM enables disentangling nucleation and growth kinetics through atomically resolved imaging of the dynamic evolution of local atomic configurations at growth sites.

The phenomenon of oxide whisker formation is not tied up with a single metal system but the oxidation of copper remains the most representative one. By employing a dedicated environmental TEM equipped with an image corrector and a differential pumping system, we demonstrate in situ TEM visualization of the growth of $\mathrm{CuO}$ nanowires during the oxidation of copper, which provides significant new insight into the rather complex kinetic process underlying the directional oxide growth [6]. By introducing oxygen gas into the sample region while simultaneously monitoring structure changes at the atomic scale, we monitor the growth of individual $\mathrm{CuO}$ nanowires by directly imaging nucleation and growth events of atomic layers at the growing end of $\mathrm{CuO}$ nanowires formed by heating metallic $\mathrm{Cu}$ in $\mathrm{O}_{2}$ gas flow (Fig. 1). Based on atomic scale observations of the upward growth at the nanowire tip, oscillatory downward growth of atomic layers on the nanowire sidewall and the parabolic kinetics of lengthening, we describe an oxide growth mechanism, driven by an axial bi-crystal boundary along the length of the nanowire. The self-propagation of atomic steps at the nanowire tip with $\mathrm{Cu}$ supplied through bi-crystal boundary diffusion enables one-dimensional crystal growth. The observed step-flow propagation of atomic layers from the nanowire tip toward the root direction further confirms the bicrystal boundary diffusion 
mechanism for the nanowire growth. The dynamic visualization of the oxide growth at the atomic scale demonstrates the remarkable ability of bi-crystal boundaries to direct the oxide growth direction and morphology. Together with density-functional theory calculations, we further show that the asymmetry in the corner-crossing barriers promotes the unidirectional oxide growth by hindering the transport of $\mathrm{Cu}$ ions from the nanowire tip to the sidewall facets. We expect broader applicability of these results in manipulating the growth of nanostructured oxides by controlling the bicrystal boundary structure that favors anisotropic diffusion for unidirectional, one-dimensional crystal growth for nanowires or isotropic diffusion for two-dimensional platelet growth [7].

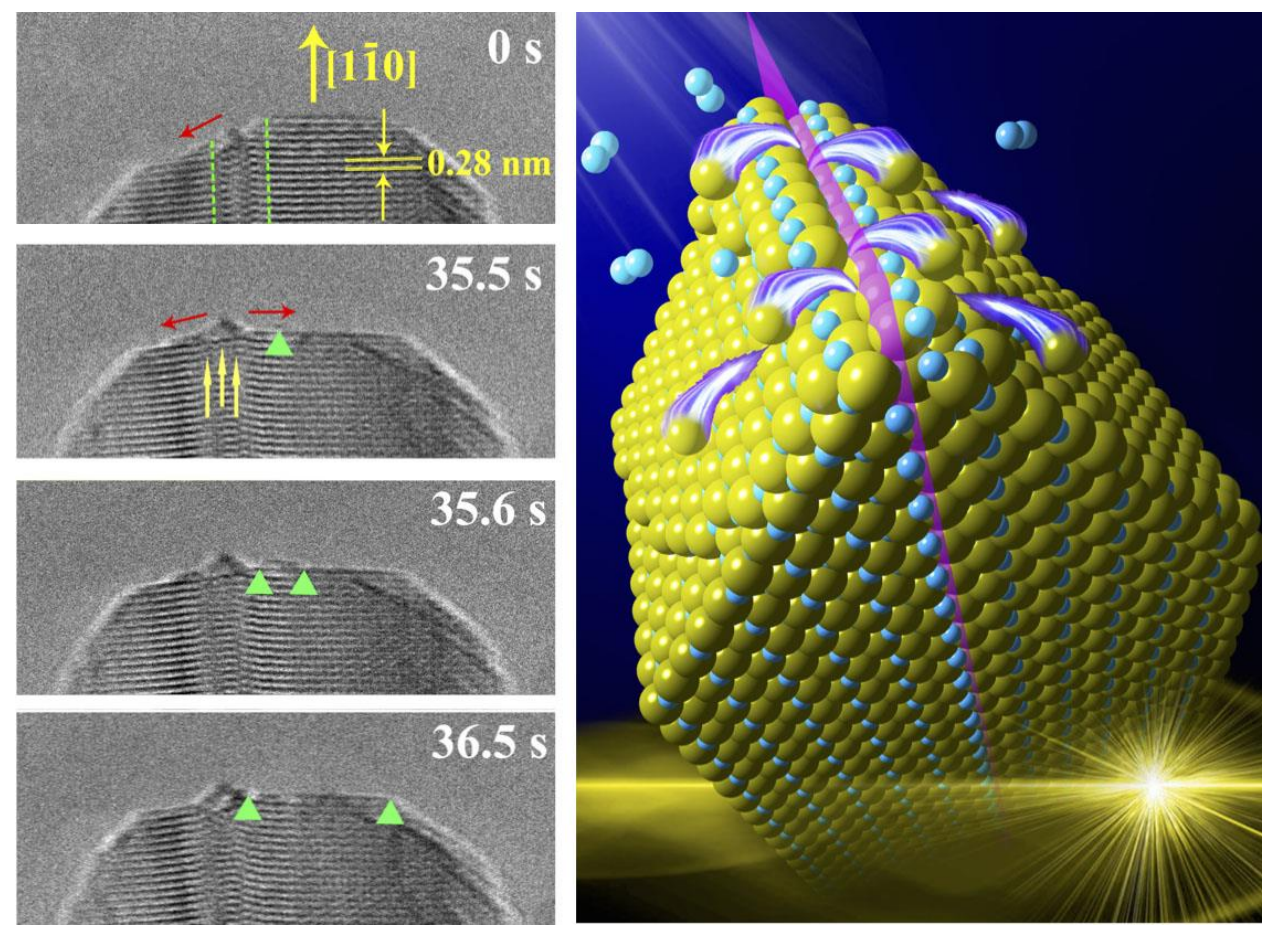

Figure 1. (Left) High-resolution transmission electron microscopy snapshots showing the tip growth of a bi-crystal $\mathrm{CuO}$ nanowire during the oxidation of copper at $400{ }^{\circ} \mathrm{C}$ and $\approx 0.5 \mathrm{~Pa}$ of $\mathrm{O} 2$ gas flow. The green triangles mark the nucleation location and subsequent step-flow growth of the atomic layer. Right: Pictorial illustration of the 1D oxide growth enabled by bicrystal grain boundary diffusion of metal ions from the nanowire root to the tip.

\section{References}

[1] Arnold, S. M., Koonce, S. E., J. Appl. Phys. 27, 964 (1956)

[2] Jiang, X.C., et. al., Nano Lett. 2, 1333 (2002)

[3] Voss, D.A., et. al., Metall. Trans. A, 13, 929 (1982)

[4] Yuan, L., et. al., Acta Mater. 59, 2491 (2011); Mater. Sci. Eng. B 177, 327 (2011); J. Mater. Res. 27, 1014 (2012); J. Electrochem. Soc. 159, C205 (2012); Materials Focus 1 (3), 222 (2012); J. Appl. Phys. 114, 023512 (2013); Nanoscale 5 (16), 7581 (2013); Chem. Phys. Lett. 590, 92 (2013); J. Cryst. Growth 390, $101(2014)$

[5] R. Mema, et. al., Chem. Phys. Lett. 512, 87 (2011)

[6] Sun, X.H., et. al., Adv. Funct. Mater. 1906504 (2019)

[7] This work was supported by the U.S. Department of Energy, Office of Sciences, Division of Materials Science and Engineering under Award No DE-SC0001135 